\title{
Adolescents with D-transposition of the great arteries repaired in early infancy demonstrate reduced white matter microstructure associated with clinical risk factors
}

\author{
Michael J. Rivkin, MD, ${ }^{\mathrm{a}, \mathrm{c}, \mathrm{d}, \mathrm{e}}$ Christopher G. Watson, ScB,${ }^{\mathrm{a}}$ Lisa A. Scoppettuolo, MS, ${ }^{\mathrm{b}}$ \\ David Wypij, PhD, ${ }^{\text {,f,i }}$ Sridhar Vajapeyam, PhD, ${ }^{\text {d,g }}$ David C. Bellinger, PhD, MSc, ${ }^{\text {a,e }}$ \\ David R. DeMaso, MD, ${ }^{\text {, c,f,h }}$ Richard L. Robertson, Jr, MD, ${ }^{\mathrm{d}, \mathrm{g}}$ and Jane W. Newburger, MD, MPH ${ }^{\mathrm{b}, \mathrm{f}}$
}

Objective: Our objective was to use diffusion tensor imaging (DTI) to compare white matter microstructure in adolescents with D-transposition of the great arteries (D-TGA) who underwent the arterial switch operation in early infancy with typically developing control adolescents. We also examined correlates between patient demographic and medical risk factors and white matter as assessed by regional fractional anisotropy (FA) values.

Methods: We used with magnetic resonance imaging (MRI) to study 49 adolescents with D-TGA and 29 control adolescents. MRI data, including whole brain DTI and conventional anatomic MRI, were acquired from each subject. Each subject's data were analyzed using random effects analysis to evaluate regional white matter differences in FA between D-TGA and control adolescents.

\begin{abstract}
Results: While multifocal punctate MRI hypointensities on T1-weighted (T1W) imaging suggestive of mineralization were found, other evidence of gross white matter injury was absent. Eighteen discrete regions of significantly reduced FA in D-TGA adolescents compared with controls were observed in deep white matter of cerebral hemispheres, cerebellum, and midbrain. Among D-TGA adolescents, lower FA correlated with younger gestational age, shorter duration of intraoperative cooling, higher intraoperative minimum tympanic temperature, longer intensive care unit stay after repair, and greater total number of open cardiac operations.
\end{abstract}

Conclusions: Despite scant white matter injury evident on conventional brain MRI, adolescents with D-TGA repaired in infancy demonstrate significant white matter FA reduction that may relate to their reported neurocognitive deficits. Among adolescents with D-TGA, FA values are associated with patient and perioperative factors, some of which are modifiable. (J Thorac Cardiovasc Surg 2013;146:543-9)

\section{Supplemental material is available online.}

Advances in prenatal diagnosis, surgical treatment, and postoperative management of children born with congenital heart disease (CHD) have dramatically improved their survival. Currently, the adult population with CHD in the United States numbers just under 3 million. ${ }^{1}$ Yet, survivors often demonstrate neurodevelopmental morbidity for which the neuroanatomic correlates remain obscure. ${ }^{2}$

\footnotetext{
From the Departments of Neurology, ${ }^{\mathrm{a}}$ Cardiology, ${ }^{\mathrm{b}}$ Psychiatry, ${ }^{\mathrm{c}}$ and Radiology, ${ }^{\mathrm{d}}$ Boston Children's Hospital; the Departments of Neurology, ${ }^{\mathrm{e}}$ Pediatrics, ${ }^{\mathrm{f}}$ Radiology, ${ }^{\mathrm{g}}$ and Psychiatry, ${ }^{\mathrm{h}}$ Harvard Medical School; and the Department of Biostatistics, ${ }^{\text {i }}$ Harvard School of Public Health, Boston, Mass.

Funded by RO1 HL77681, National Heart, Lung, and Blood Institute and the Farb Family Fund.

Disclosures: Authors have nothing to disclose with regard to commercial support.

Received for publication July 31, 2012; revisions received Nov 6, 2012; accepted for publication Dec 5, 2012; available ahead of print Feb 4, 2013.

Address for reprints: Michael J. Rivkin, MD, Department of Neurology, Pavilion 154, Boston Children's Hospital, 300 Longwood Ave, Boston, MA 02115 (E-mail: michael.rivkin@childrens.harvard.edu).

0022-5223/\$36.00

Copyright (c) 2013 by The American Association for Thoracic Surgery

http://dx.doi.org/10.1016/j.jtcvs.2012.12.006
}

Ample evidence supports a relationship between CHD and adverse consequences on the developing brain. Neuropathologic evaluation of brain in neonates who died shortly after cardiac surgery demonstrated deep white matter injury, particularly periventricular leukomalacia. ${ }^{3}$ Indeed, children whose CHD was repaired when they were neonates were more likely to have periventricular leukomalacia after surgery than those undergoing surgery when older. ${ }^{4}$

Brain magnetic resonance imaging (MRI) of infants after corrective surgery for CHD demonstrates a relationship between reduced gray matter volume and several risk factors including low preoperative cerebral blood flow, severity of perioperative hypoxia, and reduced mean blood pressure in the first postoperative day. ${ }^{5-7}$ Among a cohort of children with D-transposition of the great arteries (D-TGA), white matter injury was detected before surgical correction. ${ }^{8}$ Preoperative delayed cerebral maturation has been observed in neonates with single ventricle defects and D-TGA. ${ }^{9}$ Indeed, radiologic features of periventricular leukomalacia have been observed in term neonates with CHD before and shortly after surgery only to resolve 4 to 6 months thereafter. ${ }^{10}$ However, the possibility that alteration of white matter persists at 


$$
\begin{aligned}
& \text { Abbreviations and Acronyms } \\
& \text { BCAS }=\text { Boston Circulatory Arrest Study } \\
& \text { CHD }=\text { congenital heart disease } \\
& \text { CICU }=\text { cardiac intensive care unit } \\
& \text { D-TGA }=\text { D-transposition of the great arteries } \\
& \text { DTI }=\text { diffusion tensor imaging } \\
& \text { FA }=\text { fractional anisotropy } \\
& \text { MRI }=\text { magnetic resonance imaging } \\
& \text { ROI }=\text { region of interest } \\
& \text { SD }=\text { standard deviation } \\
& \text { T1W }=\text { T2-weighted }
\end{aligned}
$$

later ages despite resolution of these features has not yet been investigated.

The cognitive and behavioral deficits of children and adolescents born with D-TGA and treated surgically have been widely documented. ${ }^{11}$ Scant information exists about brain structural features that accompany such deficits found in homogeneous groups of children with CHD. We used diffusion tensor imaging (DTI) to compare white matter microstructure in a group of adolescents born with D-TGA, enrolled in the Boston Circulatory Arrest Study (BCAS) and corrected surgically in early infancy, with typically developing control adolescents. Further, we explored patient and medical covariates associated with white matter microstructure within the D-TGA adolescent group.

\section{METHODS \\ Subjects}

The BCAS enrolled and examined subjects with D-TGA undergoing the arterial switch procedure before 3 months of age between April 1988 and February 1992 . We ${ }^{11}$ have previously published both trial methods, as well as neurodevelopmental findings in the perioperative period and at ages 1, 4, 8 , and 16 years. For the current study, we recruited adolescents from the BCAS born with D-TGA who had undergone the arterial switch procedure before 3 months of age.

Adolescents recruited to the control group met criteria adapted from those used in the National Institutes of Health MRI study of normal brain development. ${ }^{12}$ Children with known risk factors for brain disorders (eg, intrauterine exposure to toxicants, histories of closed head injury with loss of consciousness, language disorder or Axis 1 psychiatric disorder, first-degree relative with a lifetime history of an Axis 1 psychiatric disorder, or abnormality on neurologic examination) were excluded. We excluded subjects for whom MRI was contraindicated (eg, pacemaker, metal implants), those with trisomy 21, adolescents with other forms of CHD requiring surgical correction, and subjects whose primary language was not English. This study was approved by the Boston Children's Hospital Institutional Review Board and adhered to institutional guidelines. Parents provided informed consent, and adolescents provided assent.

Image acquisition. Subjects were scanned on a GE Twin 1.5T system (General Electric, Milwaukee, Wis) at Boston Children's Hospital using a quadrature head coil. Acquisition parameters were matrix $64 \times 64$, repetition time/echo time $=13,000 \mathrm{~ms} / 108 \mathrm{~ms}$, slice thickness $=4 \mathrm{~mm}$, b-factor $=750 \mathrm{~s} / \mathrm{mm}^{2}$, number of diffusion-weighted directions $=6$, and field of vision $=240 \mathrm{~mm}$. Slices were obtained axially, aligned parallel to the anterior commissure-posterior commissure plane. Repeated acquisitions were obtained for each subject. Owing to unacceptable artifact, some subjects contributed fewer acquisitions to the analyses than others; an analysis of variance showed that there was no significant effect of series number (data not shown). Data from 55 subjects were resampled at the scanner to produce images at a resolution of $256 \times 256$. All remaining data were resampled to $256 \times 256$ using a trilinear interpolation. Examination of images before and after resampling revealed no significant difference; therefore, all images were analyzed together.

The images were first inspected by a neuroradiologist (R.L.R.) who was blinded to participant group and who used a rating form that coded information about the quality of MRI data and the presence of structural abnormality. Abnormalities were classified with respect to origin (acquired or developmental), type (infarction, mineralization, iron deposition, myelination delay, ventriculomegaly, abnormal signal characteristics), extent (focal or diffuse), and anatomic location.

Image analysis. Images were first processed with in-house software developed using the commercially available IDL programming language (http://www.ittvis.com, Boulder, Colo) to create b0, fractional anisotropy (FA), and apparent diffusion coefficient images. The b0 image provides tissue contrast similar to a conventional T2-weighted image. Next, images were analyzed using Matlab (The MathWorks, Natick, Mass) and SPM5 software (http://www.fil.ion.ucl.ac.uk/spm/) following a method similar to the optimized VBM protocol used in other published protocols using SPM5 for DTI analysis. ${ }^{13,14}$ First, b0 images were segmented into gray matter, white matter, and cerebrospinal fluid images to represent the probability that each voxel falls into 1 of the 3 tissue types. We used tissue probability maps in SPM5 provided by the International Consortium for Brain Mapping based on 452 T1-weighted (T1W) young adult images (http://www.loni.ucla.edu/ICBM/) and placed in standard Montreal Neurologic Institute space. To remove extrabrain tissue, we skullstripped all images. Only voxels exceeding a $95 \%$ probability of being gray matter, white matter, or cerebrospinal fluid were included in subsequent steps. Next, b0 images were normalized to Montreal Neurologic Institute space using parameters obtained during tissue segmentation. Subsequently, a mean image was created from the normalized b0 images. Thus, a studyspecific b0 template image was created from all control subjects and all D-TGA subjects. Next, FA images from each subject were nonlinearly registered to the template image using SPM5's Normalise function. ${ }^{15}$ Finally, the normalized FA images were smoothed with a Gaussian kernel with a full-width half-maximum of $4 \mathrm{~mm}^{3}$ to improve the signal/noise ratio.

Individual FA data sets were entered into random effects analysis in SPM5 to identify regions in which control FA was significantly greater than D-TGA FA thresholded above FA $=0.15$. The resulting statistical map was thresholded to indicate voxels surviving a $P<.0001$ (uncorrected) with a spatial cluster extent of 20 voxels or more.

Eighteen regions of interest (ROIs) demonstrated significant reduction in FA in D-TGA patients compared with controls. For each subject, the mean FA value was calculated for each ROI. Intergroup statistical analyses between D-TGAs and controls were performed using mean FA values for each ROI. Subsequently, FA values for ROIs within particular regions of brain were averaged to generate 9 composite FA values. For example, the frontal composite FA value consisted of the mean FA values derived from right frontal, right precentral, right anterior cingulate, and right insula ROIs identified in the initial analysis. Similarly, composite FA values were calculated for parietal, temporal, deep subcortical, cerebellar, midbrain, cerebellar-midbrain-deep subcortical, right hemisphere, and left hemisphere domains (see Table 1).

\section{Statistics}

Descriptive statistics included means, standard deviations, medians, and interquartile ranges as well as ranges for continuous variables, frequency counts, and percentages for categorical variables. Sociodemographic variables comprised gender, race, birth weight, gestational age at birth (in weeks), age at MRI (in years), and socioeconomic status. For D-TGA 
TABLE 1. Composite ROIs used for analysis of correlation between FA values and demographic and medical characteristics of the D-TGA group

\begin{tabular}{ll}
\hline \multicolumn{1}{c}{ Composite ROI } & \multicolumn{1}{c}{ Description } \\
\hline Frontal & $\mathrm{R}$ frontal R precentral, R anterior cingulate, \\
& $\mathrm{R}$ insula \\
Parietal & $\mathrm{R}$ parietal, L parietal \\
Temporal & $\mathrm{R}$ temporal, L temporal \\
Deep subcortical & $\mathrm{R}$ PLIC, anterior body of CC, basal ganglia \\
Cerebellar & $\mathrm{R}$ cerebellum, L cerebellum \\
Midbrain & $\mathrm{R}$ midbrain, L midbrain \\
Cerebellar-midbrain-deep & $\mathrm{R}$ cerebellum, L cerebellum, R midbrain, \\
\multicolumn{1}{c}{ subcortical } & L midbrain, R PLIC, anterior body of \\
& CC, basal ganglia \\
Right hemisphere & $\mathrm{R}$ midbrain, R cerebellum, R parietal, \\
& $\mathrm{R}$ temporal, R PLIC, \\
& $\mathrm{R}$ anterior cingulate, R precentral, \\
& $\mathrm{R}$ frontal, R insula, R basal ganglia \\
Left hemisphere & L midbrain, L cerebellum, L parietal, \\
& L temporal \\
\hline
\end{tabular}

ROI, Region of interest; $F A$, fractional anisotropy; $D-T G A$, D-transposition of the great arteries; $R$, right; $L$, left; $P L I C$, posterior limb of the internal capsule; $C C$, corpus callosum.

subjects, medical covariates measured before and during the arterial switch operation included Apgar scores at 1 minute and 5 minutes; presence of a ventricular septal defect; age at operation (>30 days vs $\leq 30$ days); and total cooling duration, total duration of deep hypothermic circulatory arrest, total support time, total time on cardiopulmonary bypass, and lowest tympanic temperature during surgery. Covariates collected in the postoperative period included history of hospital seizures (electroencephalogram or clinical), days intubated, number of days in the cardiac intensive care unit (CICU), and number of hospital days. Finally, the total numbers of cardiac operations, open operations, closed operations, and catheterizations after the arterial switch operation were considered.

Wilcoxon or Fisher's exact tests were used to compare D-TGA and control groups with respect to demographic variables and structural MRI findings. We used generalized estimating equation models assuming working independence to adjust $P$ values comparing ROIs between groups to accommodate repeated FA values per subject. Spearman correlations and linear regressions were used to analyze the associations between composite FA values and perioperative medical variables for the D-TGA patients. The Wilcoxon test was used to compare the composite FA values by categorical variables.

\section{RESULTS}

Table 2 presents the demographic and key medical characteristics of the D-TGA and control subjects from whom DTI data were acquired. Table E1 presents additional medical characteristics of the D-TGA group. In all, 49 D-TGA subjects (41 male; 8 female) who contributed 178 individual DTI data series (mean, 3.6 per subject; standard deviation $[\mathrm{SD}]=1.4)$ and 29 controls (15 male; 14 female), who contributed 99 individual DTI data series (mean, 3.4 per subject; $\mathrm{SD}=1.3$ ) were included in the final data analysis. The controls' average age was 15.0 years $(\mathrm{SD}=1.1$ years) whereas that of D-TGA patients was 16.1 years $(\mathrm{SD}=0.5$ years). Data sets of an additional 33 D-TGA and 11 controls
TABLE 2. Demographic and medical characteristics of D-TGA and control subjects from whom DTI data were acquired at 16 years of age

\begin{tabular}{|c|c|c|c|}
\hline Variable & $\begin{array}{c}\text { D-TGA } \\
(n=49)\end{array}$ & $\begin{array}{l}\text { Control } \\
(n=29)\end{array}$ & $P^{*}$ \\
\hline Birth weight, $\mathrm{kg}$ & $3.7(3.3-3.9)$ & $3.5(2.9-3.7)$ & .045 \\
\hline Gestational age, wk & $40(39.5-40)$ & $40(39-40)$ & .28 \\
\hline Male, $\%$ & 84 & 52 & .004 \\
\hline White, $\%$ & 92 & 83 & .28 \\
\hline Age at MRI, y & $16.2(16.0-16.4)$ & $14.9(14.2-16.1)$ & $<.001$ \\
\hline Social class at $16 \mathrm{y}$ of age $\dagger$ & $48(36-56)$ & $57(53-61)$ & .001 \\
\hline VSD diagnosis, $\mathrm{N}(\%)$ & $11(22)$ & & \\
\hline $\begin{array}{l}\text { Low-flow treatment group, } \\
\mathrm{N}(\%)\end{array}$ & $26(53)$ & & \\
\hline Total cooling duration, min & $15(13-20)$ & & \\
\hline Duration of DHCA, min & $41(18-56)$ & & \\
\hline $\begin{array}{l}\text { Lowest tympanic } \\
\text { temperature during } \\
\text { surgery, }{ }^{\circ} \mathrm{C}\end{array}$ & $14.5(12.2-15.5)$ & & \\
\hline ICU stay, d & $5(4-6)$ & & \\
\hline $\begin{array}{l}\text { Total No. of open } \\
\text { operations after ASO }\end{array}$ & $0(0-0)$ & & \\
\hline \multicolumn{4}{|c|}{$\begin{array}{l}\text { Values are median (interquartile range) when appropriate. } D-T G A \text {, D-transposition of } \\
\text { the great arteries; } D T I \text {, diffusion tensor imaging; } M R I \text {, magnetic resonance imaging; } \\
V S D \text {, ventricular septal defect; } D H C A \text {, deep hypothermic circulatory arrest; } \\
I C U \text {, intensive care unit; } A S O \text {, arterial switch operation. *Determined by Wilcoxon } \\
\text { or Fisher exact tests. †Score on Hollingshead Four Factor Index of Social Status, } \\
\text { with higher scores indicating higher social status. }\end{array}$} \\
\hline
\end{tabular}

were excluded owing to unacceptable signal artifact. These excluded subjects did not differ significantly from those included in the final analysis with respect to demographic characteristics of gestational age at birth, sex, race, or age at MRI acquisition. Further, they did not differ significantly from those included in the final analysis in their neurocognitive profile as defined by Wechsler Individual Achievement Tests reading and mathematics composite scores, executive function summary score, Parent Connors Attention Deficit and Hyperactivity disorder Index $\mathrm{T}$ score, Wechsler Full-Scale Intelligence Quotient (at age 8 years), and seizure occurrence.

\section{Structural Imaging Characteristics}

Subjects with D-TGA, compared with control subjects, were more likely to have an abnormality on structural MRI ( $35 \%$ vs $3 \% ; P=.002$; see Table 3 ), especially focal or multifocal abnormalities $(22 \%$ vs $0 \% ; P=.005)$. Three D-TGA adolescents and no controls had chronic focal infarction or atrophy. White matter-based, T1W punctate abnormal signal intensities indicating brain parenchymal mineralization were more prevalent in the D-TGA group ( $20 \%$ vs $0 \% ; P=.01)$. No significant differences between D-TGA and control groups existed in occurrence of diffuse, generalized, or developmental structural abnormalities.

\section{Intergroup ROI FA Difference}

We identified 18 ROIs in which mean FA values of controls significantly exceeded those of D-TGA adolescents 
TABLE 3. Structural MRI findings for D-TGA and control subjects from whom DTI data were acquired at 16 years of age

\begin{tabular}{|c|c|c|c|}
\hline Variable & $\begin{array}{c}\text { D-TGA } \\
(\mathrm{n}=49), \mathbf{n}(\%)\end{array}$ & $\begin{array}{c}\text { Control } \\
(\mathbf{n}=\mathbf{2 9}), \mathbf{n}(\%)\end{array}$ & $P^{*}$ \\
\hline Any abnormality & $17(35)$ & $1(3)$ & .002 \\
\hline $\begin{array}{c}\text { Focal or multifocal } \\
\text { abnormality }\end{array}$ & $11(22)$ & 0 & .005 \\
\hline Focal infarction or atrophy & $3(6)$ & 0 & .29 \\
\hline $\begin{array}{l}\text { Brain mineralization/iron } \\
\text { deposit }\end{array}$ & $10(20)$ & 0 & .01 \\
\hline Diffuse abnormality $\dagger$ & $2(4)$ & 0 & .53 \\
\hline Generalized abnormality & 0 & 0 & - \\
\hline Developmental abnormality $\ddagger$ & $4(8)$ & $1(3)$ & .65 \\
\hline \multicolumn{4}{|c|}{$\begin{array}{l}\text { MRI, Magnetic resonance imaging; } D-T G A \text {, D-transposition of the great arteries } \\
D T I \text {, diffusion tensor imaging. *Determined by Fisher exact tests. } \dagger \text { All diffuse abnor }- \\
\text { malities were abnormal T2 hyperintensities. } \ddagger \text { All developmental abnormalities were } \\
\text { minor malformations (Chiari malformation, enlarged perivascular space in right } \\
\text { parietal lobe, gray matter heterotopia in right frontal lobe, and right thalamic signal } \\
\text { abnormality [possible gliosis versus low-grade tumor] in the } \mathrm{D}-\mathrm{TGA} \text { group and devel } \\
\text { opmental venous anomaly in right parietal lobe in the control group). }\end{array}$} \\
\hline
\end{tabular}

(see Table 4). All images were evaluated for location of focal, multifocal, developmental, and diffuse abnormalities. None was located in any of the ROIs used for analysis of FA in both D-TGA and control groups. No ROI involved white matter at the white matter-cerebrospinal fluid interface. Areas of FA difference between the 2 groups localized to 4 brain domains: frontal, temporal, parietal, and cerebellar/midbrain (see Figures 1 and 2). All were located in deep white matter, deep to the gray matter-white matter junction. Five ROIs were located in white matter of the frontal lobes, 4 in parietal lobes, 2 in temporal lobes, 2 in cerebellum, 2 in midbrain, and 3 in deep subcortical white matter. Twelve of the ROIs were found in the right side of cerebral or cerebellar hemisphere or brainstem, and 6 were found in the left. The regions of greatest FA difference between controls and D-TGA adolescents were found bilaterally in the parietal lobes, midbrain, and cerebellum, as well as in corpus callosum.

\section{Correlation Analyses Between Composite FA Values and Demographic/Medical Variables for D-TGA Patients}

Lower mean composite cerebellar FA value correlated significantly with younger gestational age $(r=0.46$; $P=.001$ ). Similarly, both lower composite mean values for temporal and cerebellar-midbrain-deep subcortical regions tended to be associated with younger gestational age $(\mathrm{r}=0.25, P=.08 ; \mathrm{r}=0.27, P=.07$, respectively).

We also analyzed the correlation of composite FA values with perioperative medical variables. Shorter total cooling duration during the arterial switch operation was significantly associated with lower mean composite FA values of both deep subcortical and cerebellar-midbrain-deep subcortical regions $(\mathrm{r}=0.29, P=.046 ; \mathrm{r}=.31$, $P=.03$, respectively; see Figure 3); a trend toward significance was found for mean cerebellar FA $(\mathrm{r}=0.28$; $P=.052)$. Further, as the lowest tympanic temperature during surgery declined, FA rose in mean deep subcortical and mean cerebellar white matter $(\mathrm{r}=-0.32, P=.03$;

TABLE 4. White matter regions in which FA value is significantly reduced among subjects with D-TGA as compared with control subjects

\begin{tabular}{|c|c|c|c|c|}
\hline Region & Spatial coordinates (MNI) & D-TGA $(n=49)$, mean FA & Control $(n=29)$, mean FA & $\boldsymbol{P}$ \\
\hline \multicolumn{5}{|l|}{ Left hemisphere FA value } \\
\hline Parietal 1 & $(-20,-46,26)$ & 0.3571 & 0.4115 & $<.001$ \\
\hline Parietal 2 & $(-28,-40,22)$ & 0.3521 & 0.4019 & $<.001$ \\
\hline Temporal & $(-40,-14,-10)$ & 0.2030 & 0.2399 & $<.001$ \\
\hline \multicolumn{5}{|c|}{ Right hemisphere FA value } \\
\hline Parietal 1 & $(22,-46,26)$ & 0.4283 & 0.4712 & $<.001$ \\
\hline Parietal 2 & $(32,-38,22)$ & 0.4140 & 0.4439 & .006 \\
\hline Temporal & $(40,-16,-8)$ & 0.2715 & 0.3168 & $<.001$ \\
\hline Precentral & $(26,-26,52)$ & 0.3183 & 0.3638 & .014 \\
\hline Frontal 1 & $(26,18,20)$ & 0.3391 & 0.3735 & $<.001$ \\
\hline Frontal 2 & $(18,38,34)$ & 0.3416 & 0.3757 & .004 \\
\hline \multicolumn{5}{|c|}{$\begin{array}{l}\text { Subcortical, cerebellar and } \\
\text { deep white matter FA value }\end{array}$} \\
\hline Midbrain 1 (left) & $(-6,-32,-16)$ & 0.3612 & 0.4110 & $<.001$ \\
\hline Cerebellum 1 (left) & $(-22,-42,-30)$ & 0.3002 & 0.3512 & $<.001$ \\
\hline Midbrain 2 & $(8,-32,-16)$ & 0.3805 & 0.4388 & $<.001$ \\
\hline Cerebellum 2 & $(24,-38,-32)$ & 0.3743 & 0.4426 & $<.001$ \\
\hline PLIC & $(26,-18,6)$ & 0.5824 & 0.6098 & $<.001$ \\
\hline Anterior cingulate & $(14,26,26)$ & 0.2708 & 0.3089 & $<.001$ \\
\hline Insula & $(34,10,4)$ & 0.2613 & 0.2967 & $<.001$ \\
\hline Anterior body of CC & $(-2,26,14)$ & 0.3284 & 0.3928 & .003 \\
\hline Basal ganglia & $(22,-4,24)$ & 0.3137 & 0.3420 & .016 \\
\hline
\end{tabular}

FA, Fractional anisotropy; $D-T G A$, D-transposition of the great arteries; MNI, Montreal Neurologic Institute; PLIC, posterior limb of the internal capsule; $C C$, corpus callosum. 


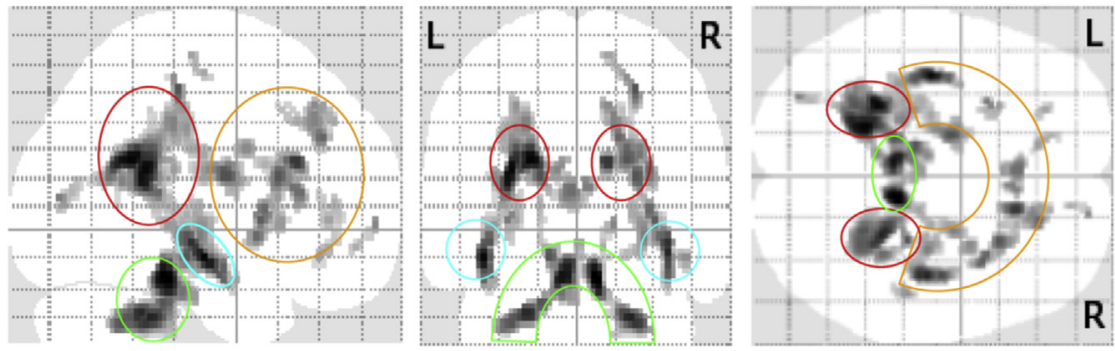

FIGURE 1. Orthogonal collapsed views of brain map for significant differences in fractional anisotropy for subjects with D-TGA versus control subjects. Note that regional differences organize into 4 regions: frontal (orange enclosure), temporal (turquoise enclosures), parietal (red enclosures), and cerebellar/ midbrain (green enclosure). $P<.001$, extent 20 voxels. $R$, Right; $L$, left; $D-T G A$, D-transposition of the great arteries.

$\mathrm{r}=-0.33, P=.02$, respectively; see Figure 3). Additionally, as the number of open operations increased, mean composite parietal FA declined $(\mathrm{r}=-0.30 ; P=.04)$. Finally, mean FA for deep subcortical white matter declined with longer CICU length of stay following the arterial switch operation $(\mathrm{r}=-0.30 ; P=.04$; see Figure 3$)$.

\section{DISCUSSION}

We found that adolescents with D-TGA repaired with the arterial switch operation in early infancy had regions of significantly lower FA, indicating abnormalities of white matter microstructure compared with control subjects of similar age. Among the adolescents with D-TGA whom we studied, lower composite FA values significantly related to younger gestational age, shorter cooling duration during the arterial switch procedure, warmer intraoperative minimum tympanic temperature, longer CICU stay after the arterial switch operation, and greater total number of open cardiac operations. The FA differences did not appear to be directly related to obvious structural abnormalities such as regional infarction or gliosis. Importantly, the white matter regions of FA reduction found among the adolescents with D-TGA harbored none of the structural abnormalities found in these subjects. Similarly, none of the punctate T1W signal abnormalities consistent with white matter mineralization, previously shown to be associated with catheterization exposure and to total bypass time during the arterial switch procedure in this cohort, were found in the identified regions of FA reduction in the D-TGA group. ${ }^{11}$

Evidence of altered white matter structure has been reported among children with CHD. Infants with D-TGA and studied with MRI have demonstrated reduced FA in

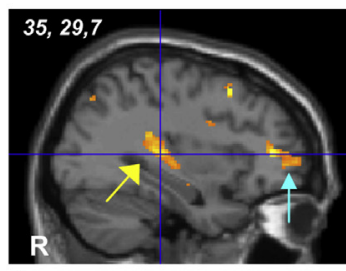

A

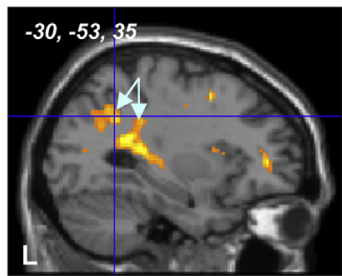

D

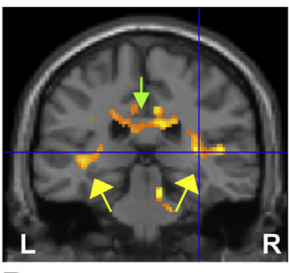

B

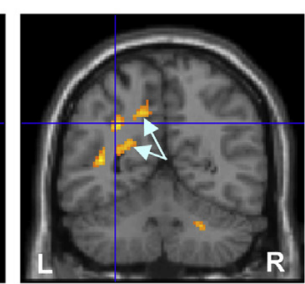

E

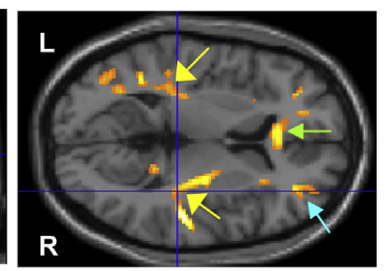

$\mathbf{C}$

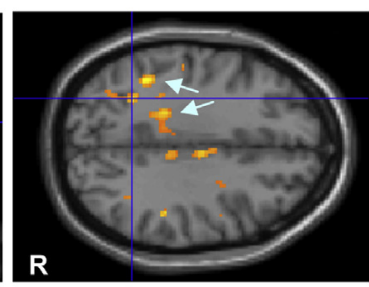

$\mathbf{F}$

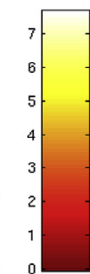

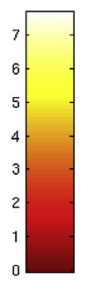

FIGURE 2. Examples of white matter regions demonstrating higher fractional anisotropy (FA) on intergroup statistical FA map for controls versus D-TGA subjects. A to $\mathrm{C}$, Orthogonal slices at 3-dimensional spatial coordinates $(\mathrm{x}=35, \mathrm{y}=29, \mathrm{z}=7)$ in the right hemisphere with cross-hairs centered on a right temporoparietal region where FA for controls exceeds that found in D-TGA subjects. Similar white matter regions of intergroup difference are seen in right frontal (turquoise arrow; A, C), anterior corpus callosum (green arrow; B, C), and superior temporal regions, bilaterally (yellow arrows; A-C). D to E, Orthogonal slices at the same 3-dimensional spatial coordinates $(x=-30, y=-53, z=35)$ in the left hemisphere with cross-hairs centered on a left parietal region where FA for controls exceeds that found in D-TGA subjects. Similar regions of intergroup difference where white matter FA measures of controls exceed significantly those of D-TGA subjects are seen throughout the parietal lobe (aqua arrows; D-F). Analyses conducted at threshold of $P<.001 ;$ cluster extent threshold of 20. Color bars provide key to $t$ scores associated with regions in which control-FA $>$ D-TGA-FA. $R$, Right; $L$, left, $D-T G A$, D-transposition of the great arteries. 
Cerebellar-midbrain-deep subcortical white matter

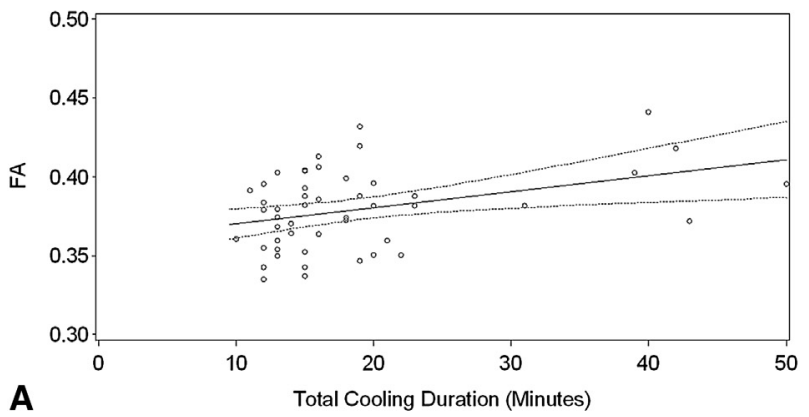

A

Deep subcortical white matter

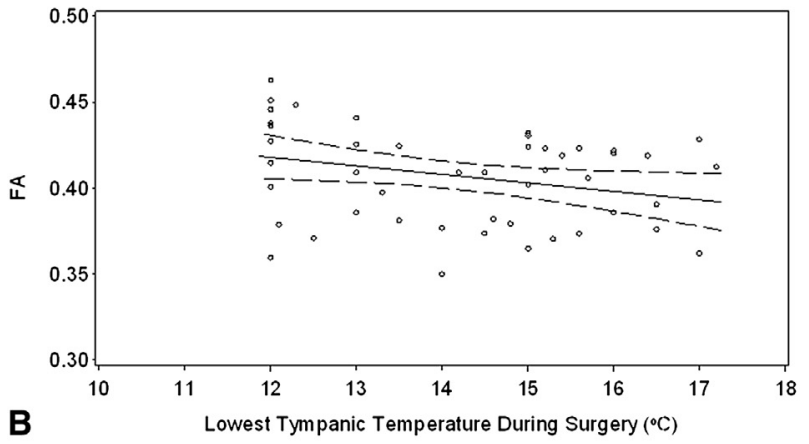

Deep subcortical white matter

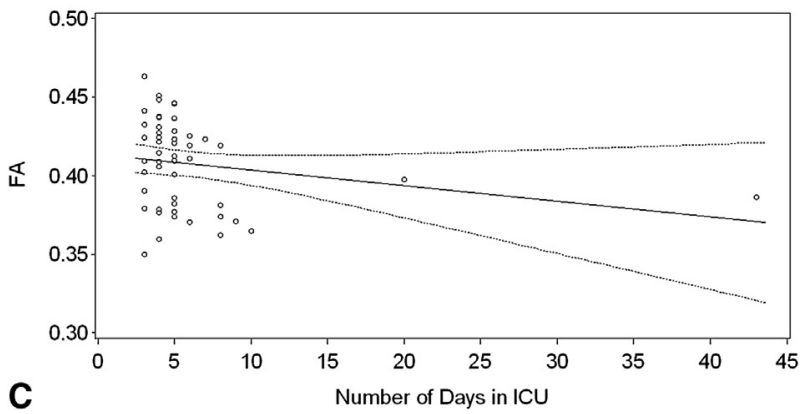

FIGURE 3. Regression lines (with $95 \%$ confidence intervals) demonstrating the correlation between FA for white matter regions and medical characteristics in the D-TGA group. A, Cerebellar-midbrain-deep subcortical white matter FA increases as total cooling duration increases (Spearman $\mathrm{r}=0.31 ; P=.03)$. B, Deep subcortical white matter FA decreases as lowest tympanic temperature during surgery increases (Spearman $\mathrm{r}=-0.32 ; P=.03)$. C, Deep subcortical white matter FA decreases as days spent in the intensive care unit increase (Spearman $\mathrm{r}=-0.30$; $P=.04) . F A$, Fractional anisotropy; $D-T G A$, D-transposition of the great arteries; $I C U$, intensive care unit.

cerebral white matter even before the arterial switch operation. ${ }^{16}$ MRI performed in newborns with CHD before and after corrective heart surgery identified white matter injury in advance of surgery in some patients, whereas in others such injury was seen only after surgery. ${ }^{6}$ Recently, a brain MRI study of adolescents with either cyanotic or noncyanotic CHD revealed white matter signal abnormality or evidence of infarct on anatomic images. ${ }^{17}$ Our study is the first to demonstrate altered deep white matter microstructure in regions without obvious white matter abnormality on routine anatomic brain MRI in adolescents with CHD.

Our data complement other pediatric publications to demonstrate that DTI may detect altered white matter microstructure in the absence of more obvious MRI signs of white matter abnormality. DTI in premature as compared with term infants revealed reduced white matter FA without evidence of injury on conventional MRI. ${ }^{18}$ Importantly, these differences persist into later life. ${ }^{19}$

Regions of reduced FA in the D-TGA group, compared with control adolescents, were located in both hemispheres and were distributed relatively symmetrically, involving frontal, parietal, and temporal lobes as well as cerebellar and pontomesencephalic regions. Overall, these regions were found in deeper white matter regions. We speculate that the regional reductions in FA contribute to recently reported cognitive deficits found in this adolescent D-TGA group. ${ }^{11}$ The spatial coordinates of the regions of FA reduction in our cohort include those reported to be traversed by superior longitudinal fasciculus, inferior longitudinal fasciculus, inferior occipitofrontal fasciculus, and uncinate fasciculus. $^{20}$ These long white matter tracts play critical roles in visual attentiveness, visual-spatial analysis, and working memory. ${ }^{20}$ Further research is required to examine how FA correlates with measured cognitive and behavioral function in children with CHD.

The etiology of FA reduction in our cohort is uncertain. Risk may accrue from genetic alterations common to CHD and brain formation or to altered fetal cerebral hemodynamics. Hypoxia, ischemia, or other stressors encountered during the prenatal/perinatal or perioperative periods could adversely affect cellular constituents of nascent cerebral tissue. Both abnormal myelin production and arrested oligodendrocyte development have been found in premature infants demonstrating reduced white matter FA. ${ }^{21}$ Additionally, diffuse axonal injury may occur across broad areas of white matter in premature infants. ${ }^{22}$ Recently, Ishibashi and associates, ${ }^{23}$ using a cardiac bypass model that included hypothermia and cardiac arrest in 3-week-old piglets, demonstrated increased proliferation of oligodendrocyte precursors, subsequent delayed oligodendrocyte maturation, and evidence of long-term axonal injury in subcortical and periventricular white matter regions. Derailment of oligodendroglial and axonal development could result in enduring compromise of white matter microstructure. Notably, many of the regions of reduced FA found in the white matter of adolescents in the D-TGA cohort we report reside in subcortical and periventricular regions of white matter.

Several risk factors correlated with altered white matter microstructure in our study have also been reported as risk factors for adverse neurodevelopmental outcome after neonatal open cardiac surgery, including younger gestational 
age, shorter cooling duration, and longer length of stay in the CICU. $^{24,25}$ In addition, we have identified higher intraoperative minimum tympanic temperature as a risk factor for altered FA in adolescents with D-TGA. Cooling duration during hypothermic cardiopulmonary bypass and intraoperative tympanic temperature represent the most easily modifiable features of care that appear to be related to white matter microstructure in our cohort. Specifically, our data suggest that longer cooling duration and lower intraoperative temperature in neonates undergoing open cardiac surgery using deep hypothermic cardiac arrest or low-flow bypass protects immature white matter and improves white matter microstructure later in life.

Our study should be interpreted in light of its limitations. The D-TGA cohort had more male subjects than the control group. Consequently, a gender-specific effect cannot be excluded. Additionally, the D-TGA group was slightly older than controls at MRI. Inasmuch as FA increases in select regions of white matter during adolescence, the slightly older age of D-TGA subjects would have tended to diminish rather than augment differences between the 2 groups. ${ }^{26}$ Third, the number of patients in our study limited the power to support multivariable analysis. Consequently, we explored associations between regional FA and subject risk factors using univariate analysis. Fourth, although we found that cooling duration and lowest intraoperative tympanic temperature were related to regional white matter microstructure measured by FA in the D-TGA group, these relationships appeared to be linear, preventing us from identifying a threshold for safe cooling. Finally, although socioeconomic status was slightly higher among control than D-TGA subjects, it was not a predictor of FA within the D-TGA group.

In conclusion, we demonstrate for the first time that adolescents with D-TGA repaired by the arterial switch operation in early infancy harbor white matter microstructure abnormalities in a multifocal pattern of FA reduction in bihemispheric cerebral deep white matter, brainstem, and cerebellum. Moreover, the severity of FA reduction is related to patient and medical factors, some of which are modifiable and potentially addressed by neuroprotective strategies.

\section{References}

1. Dearani JA, Connolly HM, Martinez R, Fontanet H, Webb GD. Caring for adults with congenital cardiac disease: successes and challenges for 2007 and beyond. Cardiol Young. 2007;17(Suppl 2):87-96.

2. Wypij D, Newburger JW, Rappaport LA, du Plessis AJ, Jonas RA, Wernovsky G, et al. The effect of duration of deep hypothermic circulatory arrest in infant heart surgery on late neurodevelopment: the Boston Circulatory Arrest Trial. J Thorac Cardiovasc Surg. 2003;126:1397-403.

3. Kinney HC, Panigrahy A, Newburger JW, Jonas RA, Sleeper LA. Hypoxic-ischemic brain injury in infants with congenital heart disease dying after cardiac surgery. Acta Neuropathol. 2005;110:563-78.
4. Galli KK, Zimmerman RA, Jarvik GP, Wernovsky G, Kuypers MK, Clancy RR, et al. Periventricular leukomalacia is common after neonatal cardiac surgery. J Thorac Cardiovasc Surg. 2004;127:692-704.

5. Watanabe K, Matsui M, Matsuzawa J, Tanaka C, Noguchi K, Yoshimura N, et al. Impaired neuroanatomic development in infants with congenital heart disease. J Thorac Cardiovasc Surg. 2009;137:146-53.

6. McQuillen PS, Barkovich AJ, Hamrick SE, Perez M, Ward P, Glidden DV, et al. Temporal and anatomic risk profile of brain injury with neonatal repair of congenital heart defects. Stroke. 2007;38:736-41.

7. Licht DJ, Wang J, Silvestre DW, Nicolson SC, Montenegro LM, Wernovsky G, et al. Preoperative cerebral blood flow is diminished in neonates with severe congenital heart defects. J Thorac Cardiovasc Surg. 2004;128:841-9.

8. Beca J, Gunn J, Coleman L, Hope A, Whelan LC, Gentles T, et al. Pre-operative brain injury in newborn infants with transposition of the great arteries occurs at rates similar to other complex congenital heart disease and is not related to balloon atrial septostomy. J Am Coll Cardiol. 2009;53:1807-11.

9. Licht DJ, Shera DM, Clancy RR, Wernovsky G, Montenegro LM, Nicolson SC, et al. Brain maturation is delayed in infants with complex congenital heart defects. J Thorac Cardiovasc Surg. 2009;137:529-36; discussion 536-7.

10. Mahle WT, Tavani F, Zimmerman RA, Nicolson SC, Galli KK, Gaynor JW, et al. An MRI study of neurological injury before and after congenital heart surgery. Circulation. 2002;106:I109-14.

11. Bellinger DC, Wypij D, Rivkin MJ, Demaso DR, Robertson RL Jr, DunbarMasterson C, et al. Adolescents with D-transposition of the great arteries corrected with the arterial switch procedure: neuropsychological assessment and structural brain imaging. Circulation. 2011;124:1361-9.

12. Evans AC. The NIH MRI study of normal brain development. Neuroimage. 2006; 30:184-202

13. Focke NK, Yogarajah M, Bonelli SB, Bartlett PA, Symms MR, Duncan JS Voxel-based diffusion tensor imaging in patients with mesial temporal lobe epilepsy and hippocampal sclerosis. Neuroimage. 2008;40:728-37.

14. Marquez de la Plata CD, Yang FG, Wang JY, Krishnan K, Bakhadirov K, Paliotta $\mathrm{C}$, et al. Diffusion tensor imaging biomarkers for traumatic axonal injury: analysis of three analytic methods. J Int Neuropsychol Soc. 2011;17:24-35.

15. Ashburner J, Friston KJ. Nonlinear spatial normalization using basis functions. Hum Brain Mapp. 1999; 7:254-66.

16. Miller SP, McQuillen PS, Hamrick S, Xu D, Glidden DV, Charlton N, et al. Abnormal brain development in newborns with congenital heart disease. $N$ Engl $J$ Med. 2007;357:1928-38.

17. von Rhein M, Scheer I, Loenneker T, Huber R, Knirsch W, Latal B. Structural brain lesions in adolescents with congenital heart disease. J Pediatr. 2011;158:984-9.

18. Berman JI, Mukherjee P, Partridge SC, Miller SP, Ferriero DM, Barkovich AJ, et al. Quantitative diffusion tensor MRI fiber tractography of sensorimotor white matter development in premature infants. Neuroimage. 2005;27:862-71.

19. Nagy Z, Ashburner J, Andersson J, Jbabdi S, Draganski B, Skare S, et al. Structural correlates of preterm birth in the adolescent brain. Pediatrics. 2009;124: e964-72.

20. Turken A, Whitfield-Gabrieli S, Bammer R, Baldo JV, Dronkers NF, Gabrieli JD. Cognitive processing speed and the structure of white matter pathways: convergent evidence from normal variation and lesion studies. Neuroimage. 2008;42: 1032-44.

21. Buser JR, Maire J, Riddle A, Gong X, Nguyen T, Nelson K, et al. Arrested preoligodendrocyte maturation contributes to myelination failure in premature infants. Ann Neurol. 2012;71:93-109.

22. Haynes RL, Billiards SS, Borenstein NS, Volpe JJ, Kinney HC. Diffuse axonal injury in periventricular leukomalacia as determined by apoptotic marker fractin. Pediatr Res. 2008;63:656-61.

23. Ishibashi N, Scafidi J, Murata A, Korotcova L, Zurakowski D, Gallo V, et al White matter protection in congenital heart surgery. Circulation. 2012;125: 859-71.

24. Goff DA, Luan X, Gerdes M, Bernbaum J, D’Agostino JA, Rychik J, et al. Younger gestational age is associated with worse neurodevelopmental outcomes after cardiac surgery in infancy. J Thorac Cardiovasc Surg. 2012;143:535-42.

25. Newburger JW, Wypij D, Bellinger DC, du Plessis AJ, Kuban KC, Rappaport LA, et al. Length of stay after infant heart surgery is related to cognitive outcome at age 8 years. J Pediatr. 2003;143:67-73.

26. Schmithorst VJ, Yuan W. White matter development during adolescence as shown by diffusion MRI. Brain Cogn. 2010;72:16-25. 
TABLE E1. Medical characteristics of subjects with D-TGA $(N=49)$ from whom DTI data were acquired at 16 years of age

\begin{tabular}{|c|c|c|c|c|c|}
\hline Variable & Minimum & 25th percentile & Median & 75th percentile & Maximum \\
\hline \multicolumn{6}{|l|}{ Preoperative characteristics } \\
\hline Apgar score at $1 \mathrm{~min}$ & 2 & 7 & 8 & 8 & 9 \\
\hline Apgar score at $5 \mathrm{~min}$ & 5 & 8 & 8 & 9 & 9 \\
\hline VSD diagnosis, $\mathrm{N}(\%)$ & & & $11(22)$ & & \\
\hline Age at operation, $\mathrm{d}$ & 2 & 4 & 6 & 9 & 61 \\
\hline Low-flow treatment group, $\mathrm{N}(\%)$ & & & $26(53)$ & & \\
\hline \multicolumn{6}{|l|}{ Operative characteristics } \\
\hline Total cooling duration, min & 10 & 13 & 15 & 20 & 50 \\
\hline Duration of DHCA, min & 5 & 18 & 41 & 56 & 72 \\
\hline Total support time, min & 116 & 133 & 140 & 156 & 203 \\
\hline Total bypass time, min & 63 & 85 & 108 & 127 & 185 \\
\hline Lowest tympanic temperature during surgery, ${ }^{\circ} \mathrm{C}$ & 12.0 & 12.2 & 14.5 & 15.5 & 17.5 \\
\hline \multicolumn{6}{|l|}{ Postoperative characteristics } \\
\hline Definite clinical or EEG seizures, $\mathrm{N}(\%)$ & & & $4(8)$ & & \\
\hline Time intubated, $\mathrm{d}$ & 1.3 & 2.0 & 2.8 & 3.9 & 28.5 \\
\hline Time in CICU, $\mathrm{d}$ & 3 & 4 & 5 & 6 & 43 \\
\hline Time in hospital, $\mathrm{d}$ & 6 & 7 & 8 & 11 & 64 \\
\hline \multicolumn{6}{|l|}{ Interim medical history after ASO } \\
\hline Total No. of cardiac operations & 0 & 0 & 0 & 0 & 3 \\
\hline Total No. of open operations & 0 & 0 & 0 & 0 & 2 \\
\hline Total No. of closed operations & 0 & 0 & 0 & 0 & 2 \\
\hline Total No. of catheterizations & 0 & 1 & 1 & 1 & 5 \\
\hline
\end{tabular}

$D$-TGA, D-transposition of the great arteries; $D T I$, diffusion tensor imaging; VSD, ventricular septal defect; $D H C A$, deep hypothermic circulatory arrest; $E E G$, electroencephalography; $C I C U$, cardiac intensive care unit; $A S O$, arterial switch operation. 